\title{
Médiévales
}

Langues, Textes, Histoire

45 | automne 2003

Grammaires du vulgaire

\section{La période de transition du latin, de la lingua romana et du français}

Traduit de l'anglais par Christopher Lucken

The Period of Transition between Latin, Lingua romana and French

\section{Roger Wright}

\section{OpenEdition}

\section{Journals}

Édition électronique

URL : https://journals.openedition.org/medievales/586

DOI : $10.4000 /$ medievales.586

ISSN : 1777-5892

\section{Éditeur}

Presses universitaires de Vincennes

Édition imprimée

Date de publication : 16 septembre 2003

Pagination : 11-24

ISBN : 2-84292-146-1

ISSN : 0751-2708

\section{Référence électronique}

Roger Wright, «La période de transition du latin, de la lingua romana et du français », Médiévales [En ligne], 45 I automne 2003, mis en ligne le 02 décembre 2005, consulté le 22 avril 2022. URL : http:// journals.openedition.org/medievales/586 ; DOI : https://doi.org/10.4000/medievales.586

Ce document a été généré automatiquement le 22 avril 2022.

Tous droits réservés 


\title{
La période de transition du latin, de la lingua romana et du français
}

\author{
Traduit de l'anglais par Christopher Lucken \\ The Period of Transition between Latin, Lingua romana and French
}

\section{Roger Wright}

1 Une langue vivante est toujours en situation de transition. Toute langue parlée change avec le temps et le latin n'a point été une exception. Aussi n'est-il guère surprenant de

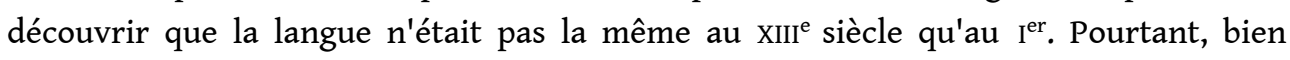
qu'elle soit fréquente, la fragmentation linguistique n'est pas une conséquence obligatoire d'un tel changement. Elle ne s'est pas produite pour le grec, qui est toujours considéré comme une seule et même langue. Elle ne s'est pas non plus produite pour l'ancien égyptien. Le latin lui-même, s'il a constamment évolué, ne semble pas s'être fragmenté en plusieurs langues durant le premier millénaire. Le fait que l'ancien français fut considéré au XIII ${ }^{e}$ siècle comme une langue distincte de l'ancien italien et de l'ancien espagnol est donc un phénomène distinct de la transition du latin au roman. Cela demande également à être étudié de près.

2 Le latin parlé s'est toujours transformé, mais toutes les composantes de cette langue n'ont pas changé au même moment ni au même rythme. Une bonne partie de son système phonologique est devenu ce que les spécialistes modernes désignent sous le nom de roman avant même la fin de l'Empire romain. Par exemple, à cette date, la distinction phonologique entre voyelles longues et voyelles brèves a disparu. Mais la plupart des éléments caractéristiques de la morphologie nominale du latin n'ont changé de manière significative qu'au cours des deux siècles suivants, époque durant laquelle la déclinaison des cas obliques des noms et des adjectifs semble avoir largement disparu de la langue parlée ; quant à la morphologie verbale et la syntaxe du latin, elles restèrent pour l'essentiel les mêmes longtemps après (et, en partie, n'ont pas changé du tout). D'anciens traits morphologiques et syntaxiques se sont maintenus durant une longue période après que leurs nouveaux concurrents furent arrivés : par exemple, les anciens temps du futur, comme cantabo, survécurent à côté des nouveaux futurs du type cantare habeo pendant de nombreux siècles, dans un état de variation 
comparable à celui qui existe en français contemporain entre «je chanterai» et «je vais chanter ». L'instant où la forme ancienne devient la variante marquée au lieu de la nouvelle (ce qui est le moment significatif lors d'un changement linguistique), cet instant ne peut être situé avec certitude. Des changements lexicaux et sémantiques singuliers surviennent constamment. Aussi n'est-il pas possible d'indiquer à quel moment exact on est passé du latin au roman. La date du septième siècle où cette transition est parfois située n'est rien de plus que le résultat d'une moyenne statistique approximative.

En réalité, les romanistes et latinistes modernes situent habituellement la transition du latin au roman au début de l'invention délibérée d'une nouvelle orthographe. C'est-àdire que les textes, de quelque époque qu'ils soient, écrits selon une orthographe non réformée - malgré les transformations de la morphologie et de la syntaxe (comme, par exemple, à certains endroits de l'ouvrage appelé le Missel de Bobbio, composé vers 700) sont considérés comme étant en latin; alors que ceux qui ont été rédigés selon l'orthographe réformée-quoique dans un registre élevé et un contexte formel (comme, par exemple, la Cantilène de sainte Eulalie) - sont considérés comme du roman. Les langues parlées changent tout le temps, mais dans une société alphabétisée (une société de litterati), les règles de la langue écrite ne changent pas avant que quelqu'un les réforme de manière volontaire. Dans une société qui utilise un système d'écriture alphabétique, de telles modernisations prennent généralement la forme d'une simple réforme orthographique. La raison en est que la morphologie, la syntaxe et le vocabulaire nouveaux peuvent être introduits de manière "naturelle » à l'intérieur de l'ancien système d'écriture, tandis qu'un trop grand écart entre les sons que l'on prononce et les signes que l'on écrit peut provoquer de temps à autre des problèmes pratiques. Dans la Gaule mérovingienne et dans l'Espagne wisigothique, le système orthographique traditionnel continuait d'être celui qui était enseigné et que l'on apprenait, mais cela ne posait pas de grandes difficultés : de nouveaux mots et de nouvelles constructions phrastiques pouvaient être utilisés à l'intérieur de ce système sans qu'il y ait besoin de le réformer. Par exemple, il n'y avait aucune difficulté pour écrire au moyen d'une orthographe non réformée la construction du complément de nom avec la préposition de suivie de l'accusatif, plutôt que d'utiliser le génitif (comme de principes au lieu de principum). Cela était favorisé par le fait que la plupart des développements syntaxiques de cette période impliquaient l'emploi intensif de mots qui avaient déjà une orthographe standard, comme illa, illorum, habes, de, plus, se, sunt, etc.; à l'évidence, des changements dans l'ordre des mots non marqués ne posaient aucun problème. De même, il n'y a aucune difficulté, au xxI siècle, pour écrire la syntaxe et le lexique du français parlé contemporain avec le système orthographique $\mathrm{du}$ français traditionnel. La seule motivation pour une quelconque réforme de l'orthographe vient d'évolutions phonétiques; mais, même là, l'écart entre la transcription phonétique et l'orthographe standard d'un mot ne pose pas de problème pour des locuteurs dont c'est la langue maternelle, qu'il s'agisse de la Gaule mérovingienne ou de la France du xxi siècle. La raison en est que la forme écrite des mots était probablement enseignée aux apprentis scribes parlant le roman de manière logographique, soit un mot après l'autre (comme pour le français contemporain) plutôt qu'une lettre après l'autre comme si l'orthographe correspondait à une écriture phonétique. 
Ce système traditionnel du latin aurait pu continuer. L'introduction d'une scripta romane n'était pas un phénomène inévitable. Il semble à présent peu vraisemblable que les règles orthographiques du français contemporain soient entièrement éliminées au profit d'une écriture phonétique, malgré le fait que l'écart, entre la forme orthographique standard et la transcription phonétique, va en s'accroissant pour de nombreux mots français. De la même manière, l'orthographe des mots du latin aurait pu se maintenir dans l'usage général, sans être réformée; le latin écrit continuera d'ailleurs à être employé longtemps après la première apparition, au $\mathrm{IX}^{\mathrm{e}}$ siècle, d'un nouveau système orthographique pour les Serments de Strasbourg, la Cantilène de sainte Eulalie et probablement d'autres textes qui n'ont pas survécu. Ce sont là les manifestations d'un nouveau système d'écriture pour une même langue, bien plus que la naissance d'une langue entièrement différente. Le roman (ou latin tardif) avait souvent été écrit auparavant, mais à l'aide du système graphique archaïque et traditionnel qui, jusqu'à un certain point, nous masque aujourd'hui son existence. Le phénomène auquel on fait parfois référence comme étant «l'essor de la scripta vernaculaire » ou «la textualisation des langues romanes », n'est rien de plus qu'un changement d'orthographe. Le roman se développait en effet depuis des siècles et avait déjà été mis par écrit à l'aide de la manière d'écrire qui avait été reçue en héritage. Le "latin tardif » et le "roman" sont donc deux noms pour la même langue. Celle-ci fut destinée à se séparer en deux courants distincts auxquels nous nous référons habituellement à travers les termes de latin médiéval et roman.

5 Comme cela est généralement reconnu, l'événement déclencheur qui a provoqué l'émergence des nouveaux systèmes d'écriture du roman tient à la purification du latin décrétée durant la Renaissance carolingienne. Les clercs de la cour de Charlemagne créèrent en effet une langue standard internationale, celle que nous appelons actuellement le latin médiéval, en demandant aux scribes de retourner à ce que les clercs estimaient être la nature archaïque du latin, aussi bien lorsqu'ils écrivaient que lorsqu'ils lisaient à haute voix. Mais leur demande était difficile à respecter. Elle mena en fin de compte le latin à devenir ce qui est appelé parfois aujourd'hui une langue "morte». D'étranges choses peuvent arriver à une langue quand elle devient une langue standard internationale; d'étranges choses peuvent aussi arriver à une langue quand elle devient une langue "morte ». Pour ce qui concerne la trajectoire du latin durant le haut Moyen Âge, ces deux destinées sont étroitement liées.

6 Bien sûr, le latin n'est pas vraiment aujourd'hui une langue «morte ». La plupart des langues qui sont actuellement décrites comme des langues «mortes» sont appelées ainsi parce qu'aucune langue parlée de nos jours ne descend d'elles directement, par exemple l'étrusque et le gaulois. Le fait est que, dans ces cas, les gens concernés choisirent progressivement de parler une autre langue. Ainsi, les descendants de ceux qui parlaient étrusque, à l'origine, choisirent de parler plutôt le latin, et les descendants de ceux qui parlaient gaulois choisirent de parler le roman. Mais le cas du latin n'est pas du tout identique. En effet, plus de $20 \%$ de la population mondiale actuelle parle une langue maternelle qui descend directement du latin. La seule raison pour laquelle nous ne pensons plus au latin comme à une langue vivante vient simplement du fait que cette langue a changé de nom. Si les langues romanes étaient toujours appelées latin de manière collective, comme le grec est toujours appelé grec (et elliniki glossa en Grèce elle-même, comme c'était déjà le cas du temps de Socrate), personne ne penserait que le latin est «mort ». Au début du IX siècle, tous les locuteurs 
de cette langue l'appelaient encore latin (lingua latina); la transition du latin au roman n'est donc, dans ce contexte, rien de plus qu'un changement de nom pour la même langue vivante.

7 Le changement de nom, de latin à roman, qui finit par se produire pour la langue parlée, fut le résultat d'une décision prise par l'élite cléricale de Charlemagne: l'état particulier du latin qui avait cours bien avant dans l'Empire romain et qui fut codifié par les grammairiens de la fin de l'Empire et leurs successeurs, devait se fossiliser sous forme de norme standard afin d'être employé comme idéal linguistique dans le cadre de l'Église. La cour carolingienne était bilingue, germanique et romane; mais ce sont les clercs qui parlaient allemand qu'il faudrait principalement blâmer pour une telle décision. La grammaire (Ars minor) de Donat était devenue la base des systèmes éducatifs de l'Antiquité tardive et la tradition pédagogique fondée sur Donat prit une très grande importance lorsque la langue latine s'étendit avec le christianisme à l'extérieur de ses frontières originaires auprès des populations de l'Europe du NordOuest qui ne parlaient pas latin. Les chrétiens d'Irlande, de Grande-Bretagne et d'Allemagne avaient donc besoin d'apprendre le latin et l'apprirent nécessairement comme une langue étrangère. Les romains eux-mêmes n'avaient aucune tradition d'un enseignement du «latin comme langue étrangère » destiné à des débutants. Donat écrivait pour des étudiants qui parlaient déjà latin et les Institutiones Grammaticae de Priscien $\left(\mathrm{au} \mathrm{VI}^{\mathrm{e}}\right.$ siècle) étaient un ouvrage de théorie linguistique de niveau universitaire tout à fait inaccessible à ceux qui ne connaissaient pas déjà bien le latin. Les clercs insulaires des $\mathrm{VII}^{\mathrm{e}}$ et $\mathrm{VIII}^{\mathrm{e}}$ siècles mirent donc au point leurs propres techniques pédagogiques. Ce qui signifiait que leur latin était essentiellement une langue écrite.

8 Les locuteurs natifs des langues celtiques et germaniques qui apprenaient le latin commençaient par l'étudier dans des textes plutôt que de recevoir un enseignement donné par des personnes dont c'était la langue maternelle. Les anciens grammairiens avaient été élevés au statut d'auctores et les clercs insulaires apprenaient à l'aide de leur auctoritas. Donat s'était concentré sur la langue écrite. Il était donc inévitable que son traité soit incomplet, dans le meilleur des cas, pour servir de guide de la langue parlée. Ce qui est normal dans la mesure où, dans toute société alphabétisée, la langue parlée inclut un certain nombre de caractéristiques que ne comprend pas la langue écrite. De plus, au IX ${ }^{e}$ siècle, de nombreux traits de la langue parlée avaient naturellement changé depuis l'époque de Donat. Aussi, une fois qu'Alcuin, élevé dans cette tradition insulaire d'un latin fondé sur des textes, fut employé par Charlemagne pour réformer le système éducatif de l'Église carolingienne, un de ses objectifs fut d'entraîner les scribes, y compris les scribes parlant roman, à lire à haute voix de cette manière artificielle et archaïque qu'il avait lui-même apprise en Northumbrie (c'est-à-dire avec un son spécifique pour chaque lettre). Telle était la fonction de son traité intitulé $D e$ orthographia, qui porte sur la prononciation lors de la lecture aussi bien que sur l'orthographe proprement dite ${ }^{1}$. C'est comme si un anglais venait ordonner aujourd'hui aux locuteurs du français contemporain de parler comme Montaigne. Aucun locuteur français ne prêterait à une telle initiative la moindre attention, bien sûr, mais Alcuin avait reçu une autorité politique et prestige sociolinguistique suffisants pour imposer une semblable exigence. Cette nouvelle grammatica du latin médiéval allait devenir, par la suite, le fondement de la Renaissance du xII ${ }^{e}$ siècle. 
9 Le roman était encore, pour l'essentiel, au viII siècle, une seule et même langue vivante. L'unité du monde romanophone, telle que lui-même la concevait, n'était pas encore divisé en deux entités séparées que les spécialistes modernes appellent désormais latin médiéval et roman. Nous pouvons être certains de la chose suivante: les écrivains de cette époque ne disent rien qui témoignerait qu'ils ont conscience de l'existence d'une semblable distinction durant la période pré-carolingienne, et il est difficile de croire que cette distinction ait pu exister sans que les locuteurs s'en soient aperçus. Par exemple, Isidore de Séville ne la mentionne pas, bien qu'il soit un écrivain encyclopédiste prolifique aussi bien qu'un linguiste compétent qui comprenait que la langue avait changé à travers les siècles. Les locuteurs romans des $\mathrm{VII}^{\mathrm{e}}$ et $\mathrm{VIII}^{\mathrm{e}}$ siècles étaient certainement conscients de l'existence de variations linguistiques, mais ils ne semblent pas avoir pensé que cela impliquait la présence de langues séparées. Les philologues modernes ont fait cette distinction à leur place, de façon anachronique.

Le point de vue de J.Herman est que le roman parlé, au viII ${ }^{e}$ siècle, était structurellement si différent du latin impérial que nous ne pouvons plus les considérer l'un et l'autre comme les étapes chronologiquement différentes d'une même langue ${ }^{2}$. Mon point de vue est que cette perspective donne trop de poids à l'aspect structural du langage (dont les implications tendent à être surestimées) et pas assez à l'aspect sociolinguistique. En effet, pour ce qui concerne l'ordre des mots, la morphologie et le vocabulaire, et si nous mettons de côté la graphie, la langue des textes composés au viII ${ }^{\mathrm{e}}$ siècle dans des régions parlant le roman est souvent aussi proche (ou plus proche) de la langue parlée au cours de ce siècle qu'elle ne l'est de celle parlée au $\mathrm{II}^{\mathrm{e}}$ siècle. Nous savons grâce aux travaux de Michel Banniard que beaucoup de ces textes, en particulier les textes hagiographiques, étaient généralement destinés à être compris quand ils étaient lus à haute voix ${ }^{3}$. Nous savons grâce aux travaux de Peter Brown que les vies de saints produisaient souvent un effet dramatique sur les auditeurs ${ }^{4}$. Nous pouvons en déduire que, dans l'ensemble, la lecture à haute voix était compréhensible, la prononciation du lector devant être suffisamment proche du roman vernaculaire pour que l'auditoire puisse reconnaître les mots. Pour l'essentiel, par conséquent, la lecture à haute voix du viII ${ }^{e}$ siècle impliquait un style de langue parlée propre à cette époque au lieu de ressembler à celui de l'Empire comme Alcuin sera amené à le préférer au siècle suivant.

11 Une distinction consciente entre le latin et le roman fut faite de temps à autre pendant le $\mathrm{IX}^{\mathrm{e}}$ siècle et ce développement métalinguistique était une conséquence des réformes carolingiennes. La moitié latine de cette nouvelle distinction est le nouveau latin médiéval plutôt que l'ancien latin tardif. La grammatica du latin médiéval, telle qu'elle fut institutionnalisée par les clercs carolingiens, dépendait pour la morphologie de l'Ars minor de Donat, qui était alors vieux de près de cinq siècles, et, pour la syntaxe, des Institutiones de Priscien, compilés à Constantinople en 527 et donc vieux de près de trois siècles. Et comme aussi bien Donat que Priscien avaient tenté à l'origine de reconstituer la grammaire écrite du passé plutôt que décrire la langue vernaculaire orale de leurs contemporains, leurs ouvrages étaient d'autant plus dépassés. Aussi, la morphologie et la syntaxe du latin enseigné aux scribes dans les écoles carolingiennes réformées étaient-elles volontairement archaïques, et, sur des points importants, bien différentes de la langue romane contemporaine.

12 Cette grammaire archaïque ne fut pas la seule conséquence malheureuse de ces nouvelles normes carolingiennes. Le nouveau latin médiéval standardisé fut encore doté, 
pour la lecture, d'une prononciation fondée sur celle employée par les Anglo-Saxons, selon laquelle chaque lettre écrite conformément à l'orthographe traditionnelle devait être pourvue d'un son particulier. À l'origine, cette pratique n'était appliquée qu'à la lecture à haute voix dans le contexte des activités ecclésiastiques. Elle était complètement différente de ce que les clercs qui parlaient cette langue vivante avaient l'habitude de faire. Jusque là, quant ils lisaient des textes à haute voix dans leur langue maternelle, les locuteurs se contentaient de prononcer les mots comme ils avaient l'habitude de le faire, omettant certains éléments tels que les lettres silencieuses, ainsi qu'on le fait dans toutes les langues quand on lit à haute voix. Cela avait l'avantage de rendre compréhensible leur lecture. L'exigence nouvelle, selon laquelle un son est attribué à chaque lettre, créa des difficultés pour les auditeurs inexpérimentés et amena les membres de l'assemblée à devenir des spectateurs passifs de la célébration liturgique plutôt que des participants actifs. (De même, si un locuteur du français contemporain essayait de lire à haute voix en prononçant chaque lettre avec le même son quelle que soit la circonstance, à la façon d'Alcuin, il aurait peu de chance de se faire comprendre.) Malgré les problèmes pratiques qui s'ensuivirent, les réformateurs carolingiens réussirent évidemment dans leur entreprise : c'est pourquoi tous ceux qui lisent aujourd'hui un texte latin à haute voix attribuent un son à chaque lettre plutôt que d'imiter la prononciation de l'auteur, même lorsqu'il s'agit de locuteurs de langues romanes lisant des textes écrits durant l'Antiquité tardive par des locuteurs parlant le roman comme Isidore de Séville ou Grégoire le Grand.

Certaines conséquences des préventions d'Alcuin contre la langue vernaculaire furent désastreuses. Elles introduisirent une division entre ceux qui connaissaient le nouveau système du latin médiéval et les autres. La conséquence sociologique la plus regrettable fut la baisse de prestige de la langue et de la culture existantes du roman, dans la mesure où les locuteurs dont c'était pourtant la langue maternelle étaient amenés à éprouver qu'ils étaient incapables désormais de la parler correctement. Aussi, dans une large mesure et pour un certain temps, leur propre culture nous devient-elle invisible. Même les érudits modernes donnent encore aujourd'hui, de temps à autre, l'impression que le roman vernaculaire n'existait pas du tout à une époque reculée. Par exemple, Michael Richter semble impliquer quelquefois que des références à des discours ou à des chants en langue vernaculaire dans des textes du haut Moyen Âge peuvent seulement se comprendre comme des références aux langues germaniques ou celtiques $^{5}$. Mais le roman était lui aussi une langue vernaculaire, même si, quand il était écrit avant les réformes $\mathrm{du} \mathrm{IX}^{\mathrm{e}}$ siècle, il prenait nécessairement une apparence ancienne.

14 Ce que nous appelons aujourd'hui latin médiéval mit longtemps avant de se répandre à travers toute l'Europe. Ce fut à partir des réformes carolingiennes qu'il devint la langue standard de la culture internationale (au sein de la respublica litterarum), employée par tous les clercs quelle que soit leur langue maternelle. Après la Renaissance du XII ${ }^{\mathrm{e}}$ siècle, le latin était même devenu une langue étrangère pour ceux dont la langue maternelle était une forme évoluée du latin. C'est ce processus de standardisation qui fit du latin une langue "morte ». Une fois qu'il était devenu une langue étrangère pour tout le monde, y compris pour les locuteurs de langue romane, le latin était «mort » au sens où, en effet, ce standard archaïque ne correspondait plus à la langue maternelle de personne. Ce processus fut essentiellement psychologique bien plus que linguistique. Quand les locuteurs du roman n'appelaient plus leur langue latin mais quelque chose 
d'autre, cela voulait dire que la conception qu'ils s'en faisaient avait changé. L'Église chrétienne s'appropria le nom de latin pour désigner son latin médiéval artificiel, cet amalgame de Donat, de Priscien, d'orthographe traditionnelle et de prononciation alphabétique conforme aux nouvelles prescriptions. Une fois la langue vernaculaire courante privée de son nom originel, le roman parlé eut besoin de trouver un autre nom pour pouvoir être identifié sans ambiguïté. D'abord, le plus souvent ce nom était tout simplement «roman » ou "romance » (avec des graphies différentes), appliqué pour commencer aux textes écrits dans de nouvelles formes. Ces termes furent modifiés plus tard à l'aide d'adjectifs géographiques, comme dans l'expression espagnole « romance castellano", ou pourvus d'un nom qui leur était propre, comme "françois ». Ainsi, participant d'un seul et même mouvement, le latin en vint à être considéré comme une langue « morte » et la langue parlée qui en était issue acquit un nom différent.

T. Janson a montré que les nouveaux noms des langues étaient tout d'abord attachés à des textes écrits dans de nouvelles formes ${ }^{6}$. Les premiers textes de cette espèce en langue romane ont été élaborés au $\mathrm{Ix}^{\mathrm{e}}$ siècle, mais ils étaient encore rares en Gaule jusqu'au XII ${ }^{\mathrm{e}}$ siècle. La langue romane écrite acquit un prestige sociolinguistique à la fin de ce siècle seulement (et en premier lieu dans les secteurs francophones de l'Angleterre). Même à cette période, de nombreux textes et documents continuaient à être écrits à l'ancienne manière, en latin. Les romanistes contemporains ne réalisent pas toujours combien les premiers textes écrits de manière délibérée en langue romane devaient apparaitre étranges à ceux qui les lisaient (comme une écriture phonétique peut le faire pour la plupart des gens). Il devait y avoir une raison déterminante pour provoquer une telle production. On peut se rendre compte maintenant que la motivation initiale à cette forme d'écriture volontairement nouvelle est une conséquence directe de la nouvelle prononciation du latin introduite par les clercs carolingiens dans le système d'éducation général. Ainsi que nous l'avons vu, ces clercs germaniques, comme les générations successives formées selon leurs exigences, lisaient des textes à haute voix en attribuant un son à chacune des lettres qui était écrite, comme si tout texte écrit était une transcription phonétique. Le fait qu'une telle réalisation était dans une grande mesure incompréhensible aux locuteurs dont la langue romane était la langue maternelle n'avait pas beaucoup d'importance à l'intérieur de l'Église, à l'exception des homélies (et celles-ci purent à nouveau, après la décision prise par le Concile de Tours en 813, être rapportées comme avant en roman afin d'être compréhensibles par tous).

Par contre, pour quelques écrits relevant d'autres genres, tels les serments, les chants religieux et certains sermons particuliers, aussi bien l'intelligibilité qu'un texte fixe étaient nécessaires. Si un locuteur de langue germanique avait besoin de lire (ou de chanter) un texte à haute voix d'une manière qui soit compréhensible pour des auditeurs de langue romane, la meilleure façon de le faire était de partir d'un texte écrit de telle sorte que les différentes lettres dont les mots étaient formés débouchent sur des sons correspondant à leur prononciation habituelle en langue romane, plutôt que sur ceux du nouveau latin standard. Une telle nécessité met sans aucun doute en valeur le système orthographique utilisé pour l'élaboration des deux premiers textes en langue romane que nous connaissons : l'un a été réalisé justement pour être lu à haute voix par un roi de langue germanique à Strasbourg (les Serments de Strasbourg), l'autre a été composé dans le but d'être chanté à haute voix par le chœur de langue germanique à Saint-Amand (la Cantilène de sainte Eulalie). Un système phonographique similaire avait 
déjà été mis en place dans les mêmes décades pour les premiers textes écrits en langue germanique. Aussi n'était-il pas nécessaire de procéder à une grande innovation technique pour étendre les mêmes principes aux textes en roman devant être lus à haute voix de façon à être compris de tous. Le nouveau système orthographique utilisé pour la langue romane se servait du même alphabet que celui que les gens éduqués connaissaient déjà. Le roman écrit n'était guère plus qu'une nouvelle scripta (employant les mêmes lettres qu'avant, mais dans de nouvelles combinaisons), plutôt qu'un alphabet entièrement neuf ou qu'une nouvelle méthode de transcription.

Avant les réformes, la lecture au sein de l'aire romane était principalement logographique, comme c est le cas en français contemporain. Par exemple, un lecteur francophone voyant la forme écrite frère dira [fré:r] et non pas [frere]. De même, en Gaule romane, quand un lecteur (un lector) de langue romane chargé de lire à haute voix identifiait dans son texte la forme écrite frater, cela lui donnait un accès direct à l'entrée lexicale correspondante de son lexique mental. Cette entrée lexicale comprenait l'information comme quoi ce mot était prononcé [frádre] : aussi était-ce ainsi qu'un lecteur l'aurait prononcé en 770. Mais un lector de langue germanique n'aurait pas procédé de cette manière, c'est-à-dire de façon logographique (un mot après l'autre), mais de façon phonographique (une lettre après l'autre). S'il voyait dans son texte la forme frater, il la lisait [frater], une forme phonétique créée par la correspondance qu'il avait appris à établir entre le son et la lettre, mais qui pouvait se révéler non reconnaissable pour un auditoire de langue romane. Comprenant que cela était susceptible de poser un problème, Nithard (ou son secrétaire), au moment d'élaborer en 842 le texte des Serments de Strasbourg, employa les mêmes correspondances entre lettres et sons, mais dans l'ordre inverse, de telle sorte que la forme qui devait être prononcée [fradre] suscita dans ce texte la forme écrite fradre. Cela devait permettre au locuteur de langue germanique de lire ce mot [fradre], plutôt que [frater] (en fait, la syllabe finale pouvait bien impliquer un schwa ${ }^{7}$ ).

Tous les mots des Serments de Strasbourg en langue romane ou germanique reçurent leur forme écrite selon ce système phonologique relativement simple. Il semble également vraisemblable que les correspondances de lettre à son étaient les mêmes pour les deux langues, ou au moins très similaires. Aussi les historiens des langues modernes peuvent-ils légitiment déduire certains traits phonétiques des langues romanes et germaniques à partir de ces Serments préparés dans ces deux nouvelles sortes de scripta.

Les circonstances dans lesquelles la Cantilène de sainte Eulalie a été élaborée quarante ans plus tard sont tout à fait semblables. Saint-Amand, comme Strasbourg, se trouvait dans une zone linguistique de transition entre les langues germaniques et romanes. Le maître de chœur de Saint-Amand, qui était alors le grand Hucbald de Saint-Amand luimême ou l'un de ses proches collaborateurs, composa la Cantilène (en relation à la sequentia latine qui se trouve dans le même manuscrit) sur des principes phonographiques similaires à ceux utilisés pour les Serments afin que le chœur, qui était de langue germanique, puisse le chanter de telle sorte à être compris par des auditeurs de langue romane. À l'évidence, le chœur chargé de chanter la séquence latine connaissait déjà ce système de correspondances entre lettres et sons ; aussi celui-ci putil immédiatement servir de modèle pour donner une forme écrite aux mots de la séquence en roman. En ce temps et en ce lieu, la langue romane vernaculaire comprenait des sons rares ou inconnus du système phonologique du nouveau latin standard, comme les diphtongues romanes écrites ou et ei dans bellezour et veintre, que 
les spécialistes modernes interprètent avec raison comme représentant les sons [ow] et [ej]. Ainsi que c'était le cas à Strasbourg, le texte en langue romane est accompagné, dans le manuscrit, d'un texte en langue germanique (le Ludwiglied) qui utilise les mêmes correspondances entre lettres et sons: par exemple, tous les deux écrivent [ts] $c z$, comme dans le mot de langue romane czo. Il est fort possible que l'habitude germanique fut ici la première et suscita une imitation en langue romane (étant donné l'absence de toute affriquée /ts/ en latin réformé).

Écrire ces textes expérimentaux était chose compliquée. Les lire devait être également difficile, comme quiconque peut s'en rendre compte s'il essaye de lire du français contemporain écrit en écriture phonétique. Ce serait une erreur de penser que l'écriture romane a été introduite pour rendre la lecture plus aisée. Pour tous ceux qui était capables de lire (les litterati), l'ancien système, qu'ils connaissaient déjà, était plus facile à utiliser, alors que, pour les illettrés, aucun système n'était plus aisé qu'un autre. En fait, le nouveau système n'était utile que pour un locuteur dont ce n'était pas la langue. Ce que nous voyons dans ces premiers textes en langue romane est donc semblable à la méthode employée aujourd'hui par les guides touristiques. Quand des locuteurs anglophones contemporains viennent en France, ils se sont souvent procuré une liste de phrases en français où les mots de cette langue sont épelés conformément aux règles de prononciation de l'anglais : par exemple, s'il vous plait est écrit see-vooplay. Un anglais lira à haute voix see-voo-play, selon les règles de correspondances phonétiques qu'il connaît, [si : vu: plej]; il pourra donc être compris (alors que s'il disait [silvousplait] il ne pourrait pas l'être). De même, à Strasbourg et à Saint-Amand, l'étranger parlant la langue germanique est amené à lire à haute voix de manière intelligible en ayant à sa disposition un texte dans lequel les mots de la langue romane vernaculaire qu'il ignore sont écrits conformément à un système de transcription phonétique qu'il connaît.

21 Puisque toutes les techniques nécessaires pour la préparation d'une nouvelle scripta impliquent une intelligence élevée et une bonne connaissance de la culture littéraire, il n'est pas surprenant de constater qu'à travers toute l'Europe romane, les premiers textes en roman ont été réalisés par des spécialistes du latin, souvent dans le même manuscrit que d'autres textes écrits en latin réformé. Ces premiers textes en langue romane ne sont donc pas des exemples d'une émancipation des illiterati, mais une forme écrite alternative préparée et utilisée par des personnes qui étaient déjà alphabétisées. Cela prendra longtemps avant que l'on apprenne à écrire et à lire les nouveaux systèmes romans sans avoir appris au préalable la bonne grammatica du latin médiéval : cela ne viendra que vers la fin du XIII ${ }^{\mathrm{e}}$ siècle.

22 La transition du roman au françois se produisit durant la même période, quatre siècles après la division initiale entre le latin médiéval et la langue romane. Le monde du huitième siècle parlant la langue romane était, sur son territoire, une communauté linguistique monolingue. À l'intérieur de l'aire parlant le roman, il y avait tout naturellement des variations linguistiques de caractère géographique, mais celles-ci étaient encore ressenties comme des variations internes à une seule et même langue. Aussi les locuteurs de langue romane pouvaient se déplacer au sein de l'aire parlant cette langue et être compris, comme peuvent le faire les locuteurs francophones actuels dans les régions du monde où l'on parle français, même s'il y a - et il y avait - des manières de parler sensiblement différentes selon les endroits et les groupes. Il semble raisonnable de penser, par exemple, qu'un visiteur de l'Espagne du Sud aurait 
probablement compris les Serments de Strasbourg s'il les avait entendus prononcer en 842. Mais, bien sûr, les formes graphiques particulières élaborées à Strasbourg et à Saint-Amand ne pouvaient servir que pour ceux qui lisaient à haute voix dans ces nouvelles régions. Ces formes écrites ne pouvaient être d'une grande utilité dans les autres aires de langue romane. C'est pourquoi, quand l'idée d'employer une scripta romane finit par s'étendre de la zone bilingue germano-romane vers les autres régions romanes, si la méthode phonographique a été généralement adoptée, les formes particulières des mots du français ne sont pas celles qui ont été adoptées ailleurs. Les italiens n'avaient aucune raison d'employer la forme écrite fradre s'ils disaient [fratéllo], et encore moins les castillans qui, pour "frère ", disaient [jermáno]. Aussi, bien que l'idée d'élaborer une nouvelle scripta romane s'étendit à partir de son origine en France du Nord, la façon précise dont elle allait se réaliser était vouée à varier d'un lieu à un autre. De la même manière, si, à l'avenir, une orthographe réformée devait être établie à partir d'une transcription phonétique de la prononciation propre aux habitants de Paris, elle ne serait pas d'une grande utilité pour les locuteurs de Marseille, de la Martinique ou du Québec. Ceux-ci risqueraient, dans ces circonstances, d'élaborer une scripta distincte de leur propre cru. Cela servirait de catalyseur pour penser que les parlers de Paris, de Marseille, de la Martinique et du Québec sont devenus des langues séparées plutôt que de continuer à être des variantes du français comme ils le sont actuellement. De même, ce fut l'extension et l'usage de nouvelles scripta romanes, différentes les unes des autres au sein de diverses régions, qui déboucha finalement sur l'idée que le français, l'occitan, le castillan, le sicilien, etc., étaient des langues distinctes plutôt que d'être simplement des variétés d'une même langue romane, ce qui était auparavant la perception générale. Cette idée, qu'il y avait plusieurs langues romanes différentes, fut donc une conséquence de l'existence de diverses scripta utilisées dans des régions séparées. Il fallut attendre jusqu'au XIII ${ }^{\mathrm{e}}$ siècle pour cela soit généralement reconnu.

Les changements linguistiques sont constants, mais, généralement, ils ne sont pas directement liés au phénomène, également intéressant mais distinct, du changement de nom d'une langue donnée. Dans les sociétés pourvues d'une culture savante, c'est habituellement l'invention de nouvelles formes écrites qui agit comme le catalyseur ultime pour changer le nom de la langue, en particulier si les nouvelles graphies que l'on prescrit sont différentes dans les diverses zones d'un ancien continuum linguistique. Dans le cas du roman, la nécessité d'un nouveau système graphique n'existait pas avant que les réformes archaïques de la prononciation latine ne soient introduites à partir des régions germaniques de la chrétienté. L'établissement d'une distinction conceptuelle entre le latin médiéval et le roman, ainsi que la fragmentation qui s'en est suivi pour ce qui concerne ce dernier, partagé désormais entre le françois et les autres langues romanes, ont été les conséquences des réformes carolingiennes. Ce qui explique pourquoi ces transitions orthographiques commencèrent au moment où celles-ci eurent lieu ${ }^{8}$. 


\section{NOTES}

1. Cf. Alcuin, De Orthographia, S. Bruni éd., Florence, 1997.

2. Cf. J. Herman, Le Latin vulgaire, Paris, 1965 , Du latin aux langues romanes. Études de linguistique historique, réunies par S. Kiss avec une préface de J. Monfrin, Tübingen, 1990, ainsi que la trad. anglaise largement augmentée du premier de ces deux ouvrages, Vulgar Latin, R. Wright trad., Philadelphie, 2000.

3. Cf. M. Banniard, Viva voce. Communication écrite et communication orale $d u I V^{e}$ au IX siècle en Occident latin, Paris, 1992, Genèse culturelle de l'Europe, $V^{e}$-VIII ${ }^{e}$ siècles, Paris, 1989, " Latin tardif et français prélittéraire : observations de méthode et de chronologie ", Bulletin de Société Linguistique de Paris, 88, 1993, p. 139-62, et « Changement de phase et changement de langue ( $\mathrm{VII}^{\mathrm{e}} / \mathrm{VIII}^{\mathrm{e}}$ siècles) en occident latin ", dans De Lingua Latina Novae Quaestiones, C. Moussy éd., Louvain, 2001, p. 1021-31.

4. Cf. P. BRown, The Cult of the Saints : Its Rise and Function in Latin Christianity, Chicago, 1982.

5. Cf. M. RICHTER, The Formation of the Medieval West: Studies in the Oral Culture of the Barbarians, Dublin, 1994.

6. Cf. T. JANSON, Speak: a short history of languages, Oxford, 2002.

7. Cf. G. Hilty, « Les Serments de Strasbourg et la Séquence de sainte Eulalie », Vox Romanica, 37, 1978, p. 126-50.

8. Pour de plus amples réflexions sur la transition du latin au roman, voir R. Wright, Late Latin and Early Romance in Spain and Carolingian France, Liverpool, 1982, et A Sociophilological Study of Late Latin, Turnhout, 2003, ainsi que les différentes études recueillies dans Latin and the Romance Languages in the Early Middle Ages, R. Wright éd., Londres, 1991 (reprint : Penn State, 1996), et dans La transizione dal latino alle lingue romanze, J. Herman éd., Tübingen, 1998. Pour les îles britanniques, voir V. LaW, The Insular Latin Grammarians, Woodbridge, 1982, et Grammar and Grammarians in the Early Middle Ages, Londres, 1997. Dans une perspective moins linguistique, voir J. Farrell, Latin Language and Latin Culture, from Ancient to Modern Times, Cambridge, 2001. Pour le XIII siècle, voir également $\mathrm{R}$. Wright, El Tratado de Cabreros (1206) : estudio sociofilológico de una reforma ortográfica, Londres, 2000, notamment les chap. 1 et 4. Voir enfin R. Balibar, L'Institution du français. Essai sur le colinguisme des Carolingiens à la République, Paris, 1985, B. Cerquiglini, La Naissance du français, Paris, 1991, et R. A. Lodge, Le Français. Histoire d'un dialecte devenu langue, Paris, 1997 [1993].

\section{RÉSUMÉS}

La langue vivante encore appelée lingua latina au VIII ${ }^{\mathrm{e}}$ siècle était alors conçue comme une seule langue malgré sa grande variation sociale et géographique. À la fin du XIII siècle, cependant, elle s'était divisée en plusieurs langues: le français, la langue d'oc, le catalan, le castillan, le portugais, l'italien, etc., de même que la langue «morte » connue aujourd'hui sous le nom de 
latin médiéval. La première étape de ce processus apparut au IX $\mathrm{I}^{\mathrm{e}}$ siècle dans le royaume carolingien quand, pour la première fois, on établit une distinction systématique entre le latin (une grammatica standardisée que nous appelons latin médiéval) et le roman. Ce type de latin fut standardisé dans l'écrit selon les règles de Donat et de Priscien, et dans l'oral selon l'usage des clercs de langue germanique qui avaient l'habitude de prononcer toutes les lettres comme s'ils les lisaient à haute voix, avec un son spécifique pour chaque lettre. La moitié romane de cette dichotomie naissante combinait l'usage oral avec une nouvelle forme écrite, initialement établie (par exemple, dans la Cantilène de sainte Eulalie) en attribuant un symbole écrit à chaque son prononcé, en se fondant sur les correspondances entre lettres et sons instaurées par les réformateurs carolingiens pour le latin médiéval. Avant le $\mathrm{XI}^{\mathrm{e}}$ siècle, cette nouvelle écriture du roman était pour l'essentiel une forme d'écriture expérimentale; par la suite, les différentes régions parlant une langue romane devaient épeler les mots de diverses façons, et l'idée qu'il y avait différentes langues romanes plutôt qu'une seule fut généralement acceptée. Une telle transition est donc une question aussi bien de changement linguistique que de changement de nom de la langue, ce dernier point étant une question de politique tout autant que de linguistique.

The Period of Transition between Latin, Lingua romana and French. The living language which was still called lingua latina in the eighth century A.D. was thought of then as being one single language despite wide social and geographical variation. But it had split into several languages by the end of the thirteenth century : French, Provenzal, Catalan, Castilian, Portuguese, Italian, etc., as well as the «dead» language we know of as Medieval Latin. The initial stage in this process came in the Carolingian realms during the ninth century, when for the first time some people made a systematic distinction between Latin (standardized grammatica, what we would call Medieval Latin) and Romance. This kind of Latin was standardized in writing according to the rules of Donatus and Priscian, and in speech according to the procedures of the Germanic-speaking scholars who were used to pronouncing all words as if reading them aloud, with a specified sound for each written letter. The Romance half of the incipient dichotomy combined the spoken register with a new written form, initially based (e.g. in the Cantilène de sainte Eulalie) on providing a written symbol for each spoken sound roughly according to the correspondences between letter and sound that had been established by the Carolingian reformers for Medieval Latin. This new written Romance form was essentially an experimental way of writing before the eleventh century ; after that, different Romance-speaking areas needed to spell words in different ways, and the idea that there were several Romance languages rather than one became generally accepted. Thus the transitions are a question both of language change and of change of the language's name, and the latter is as much a question of politics as it is of linguistics.

\section{INDEX}

Mots-clés : Cantilène de sainte Eulalie, langue romane, latin médiéval, Serments de Strasbourg, sociolinguistique

Keywords : late Latin, romance, sociolinguistics

\section{AUTEUR}

\section{ROGER WRIGHT}

University of Liverpool, Department of Hispanic Studies, Liverpool, G.-B.-L69 3BX 